\title{
Post-procedural Bacillus cereus septic arthritis in a patient with systemic lupus erythematosus
}

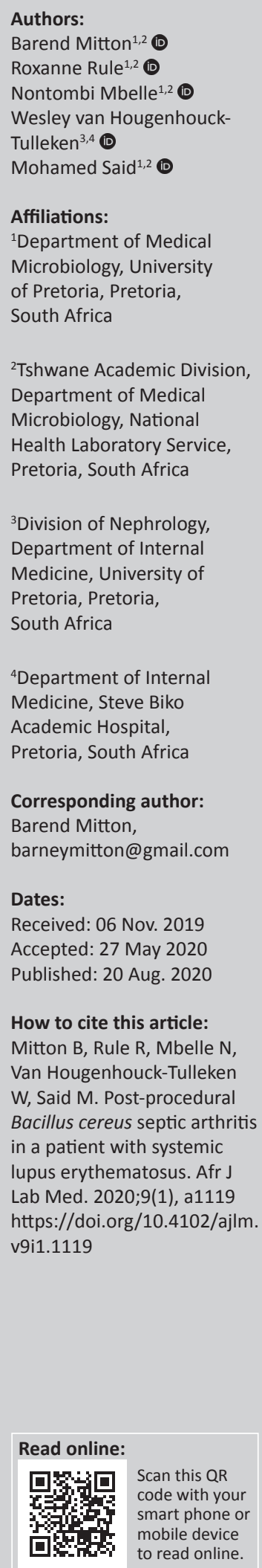

Introduction: Bacillus species are often considered as contaminants when cultured from clinical samples. Bacillus cereus may be a pathogen in certain circumstances and is known to cause musculoskeletal infections. This report aims to educate clinicians and clinical microbiology laboratories on B. cereus musculoskeletal infections and to heighten awareness that Bacillus species should not always be dismissed as contaminants.

Case presentation: We report the case of a patient who presented to a tertiary hospital in Pretoria, South Africa, in November 2018 with B. cereus septic arthritis and underlying systemic lupus erythematosus (SLE). The isolate would otherwise have been dismissed as a contaminant had it not been for the crucial interaction between the laboratory and the treating clinicians. To our knowledge, this is the first case report of septic arthritis caused by B. cereus in an SLE patient where the organism was cultured from the joint specimen. Identification of the organism was performed using matrix-assisted laser desorption/ionisation mass spectrometry.

Management and outcome: Definitive treatment was with intravenous vancomycin, continued for four weeks, in addition to arthroscopy and management of the underlying SLE. The patient had a good clinical outcome and regained full mobility.

Conclusion: Musculoskeletal infections, specifically septic arthritis caused by B. cereus, are exceedingly rare infections. Immune suppression, trauma, prosthetic implants and invasive procedures are important risk factors for $B$. cereus musculoskeletal infections. Close collaboration with a multi-disciplinary team approach will effect the best outcome for complicated patients with B. cereus infections.

Keywords: Bacillus cereus; septic arthritis; systemic lupus erythematosus; Matrix-assisted laser desorption/ionisation mass spectrometry; MALDI-TOF MS; musculoskeletal infection; arthroscopy.

\section{Introduction}

As the vast majority of Bacillus species are non-pathogenic and ubiquitous in the environment, many clinical microbiologists and clinicians dismiss Bacillus species cultured from clinical specimens as contaminants. Occasionally, this results in a missed diagnosis and inappropriate clinical decision-making. This case is important, because it illustrates the importance of communication between clinicians and the clinical laboratory staff in determining the significance of culture results. This report aims to educate healthcare workers on Bacillus cereus joint infections and further endeavours to assist healthcare practitioners in distinguishing when this organism should be dismissed as a contaminant and when it should be considered as a pathogen.

\section{Ethical considerations}

Written, informed consent was obtained from the patient. This research was approved by the University of Pretoria, Faculty of Health Sciences, Research Ethics Committee (ethics reference number 133/2019).

\section{Case presentation}

In November 2018, a 32-year-old male was referred to a tertiary academic hospital in Pretoria, South Africa, with a non-resolving septic arthritis of his right knee. The patient presented to a secondary hospital 10 days prior with a tender, swollen right knee, with no history of trauma. He underwent an arthroscopy at that centre and received intravenous amoxicillin-clavulanic acid, with a suboptimal response. In addition, he developed symptoms suggestive of systemic lupus erythematosus (SLE),

Copyright: (C) 2020. The Authors. Licensee: AOSIS. This work is licensed under the Creative Commons Attribution License. 
which included polyarthritis, xerostomia, Raynaud's phenomenon, proteinuria and confusion. On examination he was haemodynamically stable with a pulse rate of 100 beats per minute, a blood pressure of $117 / 81 \mathrm{mmHg}$ and a temperature of $36.5{ }^{\circ} \mathrm{C}$. He had asymmetric polyarthritis, involving the right elbow, left wrist, right knee and both ankles. The right knee was the worst affected, with swelling, erythema and tenderness on examination. An emergency arthroscopy of the right knee was performed, revealing a purulent effusion. Intravenous ceftriaxone ( $1 \mathrm{~g}$ twice daily) was started empirically. X-rays of all affected joints revealed no accompanying osteomyelitis. No other imaging of the joints was done. The diagnosis of SLE was confirmed based on a Systemic Lupus International Collaborating Clinics score ${ }^{1}$ of five (anti-nuclear antibody positive, lupus nephritis class 3, arthritis, low C3 and neurologic SLE).

\section{Laboratory investigations}

Admission blood test revealed a white cell count of $8.04 \times 10^{9}$ cells / L with neutrophilia (73\%), a C-reactive protein of $107 \mathrm{mg} / \mathrm{L}$, a positive anti-nuclear antibody (titre 160) and a low C3 (0.50 g/L). In addition, an Epstein-Barr virus viraemia of 530 copies $/ \mathrm{mL}$ was found. Admission blood cultures had no growth. A pus sample taken during arthroscopy showed numerous Gram-positive bacilli on the direct Gram stain. Culture revealed large, flat, grey, beta-haemolytic colonies on $5 \%$ horse blood agar (Figure 1), which also grew on chocolate agar and MacConkey agar. This isolate was identified as Bacillus species and reported as a possible contaminant. The patient had a poor clinical response to the empiric ceftriaxone after 6 days of treatment, and a request was made by the attending clinicians for antimicrobial susceptibility testing on the isolate. The isolate was therefore referred for further identification to species level using matrix-assisted laser desorption/ionisation mass spectrometry by means of VITEK $^{\circledR}$ MS (bioMérieux, Marcy l'Etoile, France), instrument software version 1.5.0.4, MYLA ${ }^{\circledR}$ version 4.5.1 (bioMérieux), Knowledge Base (database) version 3.2. The isolate was identified as Bacillus cereus. Antibiotic susceptibilities were performed by ETEST $^{\circledast}$ (bioMérieux, Marcy l'Etoile, France) and interpreted using the Clinical \& Laboratory Standards Institute M45 (2015) breakpoints. ${ }^{2}$ The isolate was resistant to penicillin (minimum inhibitory concentration [MIC] $32 \mu \mathrm{g} / \mathrm{mL}$ ), and cefotaxime (MIC $32 \mu \mathrm{g} / \mathrm{mL}$ ), but susceptible to vancomycin (MIC $4 \mu \mathrm{g} / \mathrm{mL}$ ) and imipenem (MIC $4 \mu \mathrm{g} / \mathrm{mL}$ ).

\section{Management and outcome}

Based on the report, the patient was started on intravenous vancomycin $1 \mathrm{~g}$ twice daily. The dose was adjusted to achieve a target vancomycin blood trough level of $15 \mathrm{mg} / \mathrm{mL}-20 \mathrm{mg} /$ $\mathrm{mL}$. Trough levels were monitored roughly every 3 days over the treatment period, and the dosage adjusted accordingly. No vancomycin-related adverse events occurred during this time. Vancomycin was continued for 4 weeks; over this period, the pain and swelling improved dramatically, inflammatory markers normalised and the patient regained mobility. Management of the SLE included prednisone, mycophenolate mofetil and chloroquine, with good response.

\section{Discussion}

Bacillus species are Gram-positive, aerobic or facultative anaerobic sporulating bacilli, which are ubiquitous in the environment. ${ }^{3}$ Over 100 species are known to belong to the genus. ${ }^{3}$ Bacillus cereus is a common cause of food poisoning and occasionally causes opportunistic infections, usually in vulnerable hosts. These infections include ophthalmic infections, wound infections, septicaemia, endocarditis, meningitis, necrotising pneumonia and orthopaedic infections. ${ }^{3,4}$ Important virulence factors of B. cereus include production of toxins and the formation of biofilms and spores. ${ }^{4}$ Except for B. cereus and B. anthracis, the genus Bacillus is rarely associated with disease..$^{3,5,6,7}$ Therefore, Bacillus species are often reported as contaminants, even when cultured from sterile specimens. This may result in a delay in correct diagnosis and inappropriate treatment.

Bacillus cereus grows well on most routine culture media such as blood agar (Figure 1), chocolate agar and MacConkey agar. Routine laboratory tests will reveal large boxcar-shaped, Gram-positive bacilli (Figure 2) that are catalase positive. However, identification to species level requires specialised techniques such as matrix-assisted laser desorption/ionisation mass spectrometry or molecular techniques. $^{7}$

Musculoskeletal infections caused by $B$. cereus are rare but have been previously reported in literature. ${ }^{4,5,6,7}$ Åkesson, Hedströum and Ripa. ${ }^{4}$ reported 12 cases of B. cereus orthopaedic infections, all occurring in post-operative or post-traumatic wounds, whilst Dubouix et al. $^{5}$ reported 41 cases with $B$. cereus wound infections associated with open fractures. Gallo et al. ${ }^{6}$ reported two cases of B. cereus prosthesis-related septic arthritis, which were culture negative but identified using PCR-mass-spectrometric-technology and fluorescence

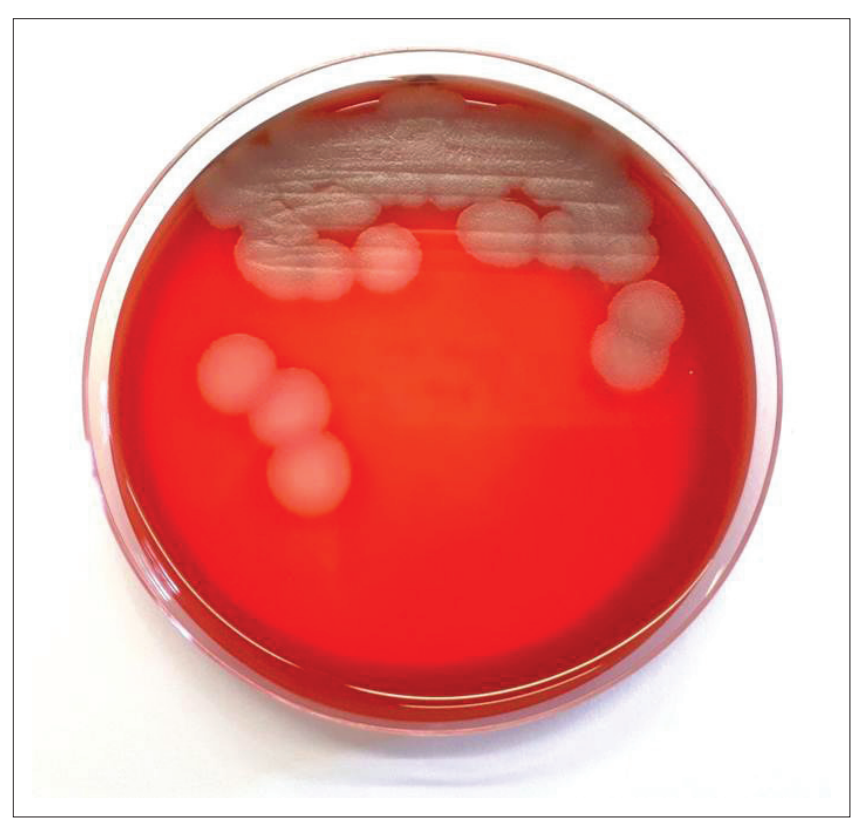

FIGURE 1: Typical morphology of Bacillus cereus on 5\% horse blood agar (Pretoria, South Africa, 19 March 2019). 


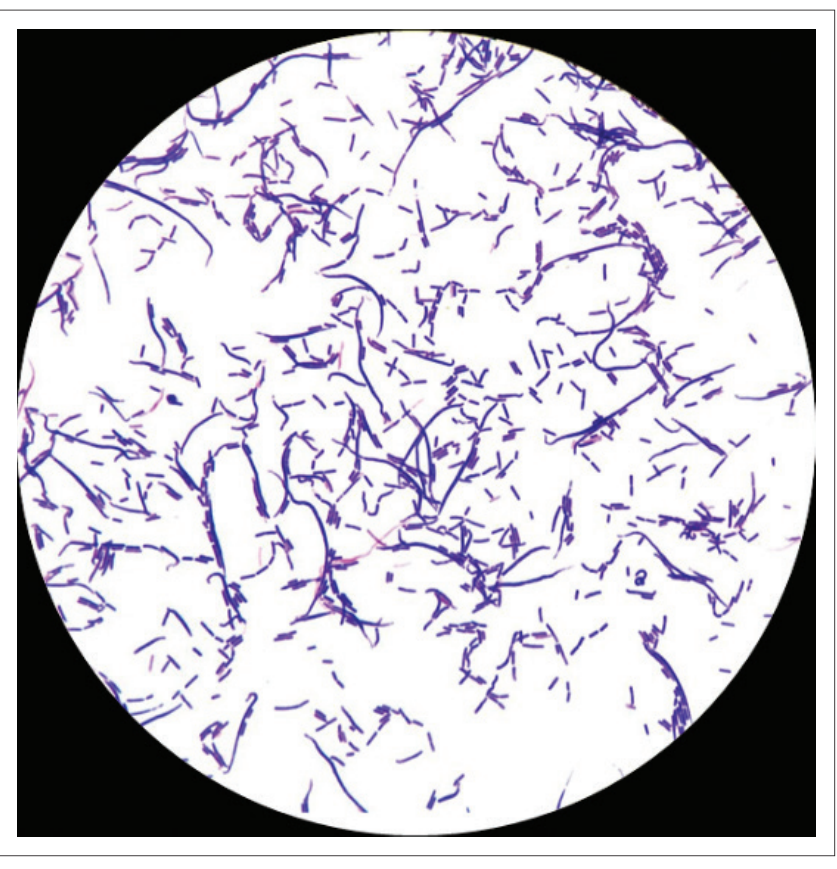

FIGURE 2: Microscopic image at $1000 \times$ magnification showing boxcarshaped, Gram-positive bacilli, typical of Bacillus cereus (Pretoria, South Africa, 19 March 2019).

in situ hybridisation of tissue. Ha et al. ${ }^{7}$ reported a single case of late prosthetic joint infection with $B$. cereus that occurred 13 years after total hip replacement surgery, confirmed by $16 \mathrm{~S}$ ribosomal ribonucleic acid (rRNA) sequencing. In total, we found 56 cases reported over 25 years, highlighting the rarity of $B$. cereus musculoskeletal infections and emphasising the difficulty in making a definitive diagnosis. The scarcity of these infections may actually be the result of under-reporting, as Bacillus species are often reported as contaminants.

Bacillus cereus produces $\beta$-lactamases and is resistant to most $\beta$-lactam antibiotics, except carbapenems. ${ }^{3}$ Classically, Bacillus species are susceptible to vancomycin, aminoglycosides and fluoroquinolones, whilst resistance to erythromycin, tetracycline and even carbapenems have been reported. ${ }^{3}$ The empiric antibiotic of choice for invasive $B$. cereus infections is vancomycin. ${ }^{3,7}$ In addition to administering antibiotics, it is imperative to obtain source control at the infected site, as B. cereus is known to form biofilm. The removal of implanted medical devices may be necessary.?

The concomitant SLE may be considered a notable risk factor for invasive $B$. cereus infection in this patient. It is uncertain if the Epstein-Barr virus viraemia played a significant role in predisposing the patient further to this infection. However, both SLE and Epstein-Barr virus infection are known to be immune-modulatory, down-regulating the innate and humoral systems and placing the patient at increased risk of opportunistic infections. ${ }^{8,9}$ An additional risk factor was the preceding arthroscopy, which may have introduced spores into the joint space. To our knowledge, this is the first case of septic arthritis in an SLE patient where the organism was cultured from the joint specimen. The communication between the clinical and microbiology teams ensured that the organism was identified to species level and that antibiotic susceptibility testing was performed, resulting in a favourable outcome for the patient.

\section{Conclusion}

Bacillus species are often regarded as contaminants and receive little attention from the medical community. In certain highrisk patient groups, however, $B$. cereus may be a formidable pathogen. Clinical microbiology laboratorians and clinicians should have a high index of suspicion in these patients and identify Bacillus species in cultures from sterile sites to species level as well as perform antibiotic susceptibility when B. cereus is identified. Immune suppression, trauma, prosthetic implants and invasive procedures are important risk factors for B. cereus musculoskeletal infections. Bacillus cereus is universally resistant to most $\beta$-lactam antibiotics, with the exception of carbapenems. Treatment with vancomycin was successful in the case described. Close collaboration with a multi-disciplinary team approach will effect the best outcome for complicated patients with B. cereus infections.

\section{Acknowledgements Competing interests}

The authors have declared that no competing interests exist.

\section{Authors' contributions}

B.M. designed the data collection tools, collected case data, analysed the data, and drafted and revised the paper. B.M. was also the guarantor. R.R. collected case data, and drafted and revised the paper. N.M. drafted and revised the paper. W.v.H-T. collected case data, analysed the data, and drafted and revised the paper. M.S. designed the data collection tools, analysed the data, and drafted and revised the paper.

\section{Sources of support}

This research did not receive any specific grant from funding agencies in the public, commercial or not-forprofit sectors.

\section{Data availability statement}

Data sharing is not applicable to this article as no new data were created or analysed in this study.

\section{Disclaimer}

The views and opinions expressed in this article are those of the authors and do not necessarily reflect the official policy or position of any affiliated agency of the authors.

\section{References}

1. Petri M, Orbai A-M, Alarcón GS, et al. Derivation and validation of the Systemic Lupus International Collaborating Clinics classification criteria for systemic lupus erythematosus. Arthritis Rheum. 2012;64(8):2677-2686. https://doi.org/10.1002/ art.34473 
2. Clinical and Laboratory Standards Institute (CLSI). Methods for antimicrobial dilution and disk susceptibility testing of infrequently isolated or fastidious bacteria. 3rd ed. CLSI guideline M45. Wayne, PA: Clinical and Laboratory Standards Institute; 2015.

3. Bottone EJ. Bacillus cereus, a volatile human pathogen. Clin Mircobiol Rev. 2010;23(2):382-398. https://doi.org/10.1128/CMR.00073-09

4. Åkesson A, Hedströum SÅ, Ripa T. Bacillus cereus: A significant pathogen in postoperative and post-traumatic wounds on orthopaedic wards. Scand J Infect Dis. 1991;23(1):71-77. https://doi.org/10.3109/00365549109023377

5. Dubouix A, Bonnet E, Alvarez M, et al. Bacillus cereus infections in traumatology Orthopaedics department: Retrospective investigation and improvement of healthcare practices. J Infect. 2005;50(1):22-30. https://doi.org/10.1016/j.jinf.2004.05.012
6. Gallo PH, Melton-Kreft R, Nistico L, et al. Demonstration of Bacillus cereus in orthopaedic-implant-related infection with use of a multi-primer polymerase chain reaction-mass spectrometric assay: Report of two cases. J Bone Joint Surg. 2011;93(15):e85. https://doi.org/10.2106/JBJS.J.01181

7. Ha J, Park YJ, Kim YJ, Oh HC. Late prosthetic joint infection and bacteremia by Bacillus cereus confirmed by $16 \mathrm{~S}$ rRNA sequencing and hip joint tissue pathology. Ann Clin Microbiol. 2016;19(2):54-57. https://doi.org/10.5145/ACM.2016.19.2.54

8. Ning S. Innate immune modulation in EBV infection. Herpesviridae. 2011;2(1):1. https://doi.org/10.1186/2042-4280-2-1

9. Tikly M, Navarra SV. Lupus in the developing world - Is it any different? Best Pract Res Clin Rheumatol. 2008;22(4):643-655. https://doi.org/10.1016/j.berh.2008. 05.003 